Background In September 2012, an anticipatory prescribing policy, the 'Just in Case Four Core Drugs' (JiC4CD) was introduced across the healthcare economy after a multidisciplinary group of clinical experts reviewed current practices. The JiC4CD policy seeks to deliver optimal care to patients with palliative illness in the community by:

supporting patient choice if they wish to remain at home avoiding distress caused by poor access to medicines, by anticipating need

avoiding the distress caused to patients, carers and healthcare staff due to not having the correct medicine readily available and any attendant delays in achieving symptom control reducing inappropriate admissions

Anticipatory drugs are dispensed for patients thought to be in the last four weeks of life who are being discharged from hospital using the fast-track discharge process. The availability of JiC4CD in the home support proactive symptom control by promoting anticipatory prescribing.

Aims To identify the number of patients who received JiC4CD upon discharge from Blackpool Victoria Hospital and compare with the number of fast-track discharges.

Method Electronic discharge prescriptions over a 12-month period were analysed JiC4CD and the figures were compared with the number of fast-track discharges.

Results A total of 211 discharges for JiC4CD were dispensed by the hospital pharmacy over the 12 month period. There were 227 fast-track discharges during the same period.

Conclusion The study showed that $93 \%$ of fast-track discharges received JiC4CD. Ideally, all patients discharged utilising the fast-track discharge policy should receive JiC4CD. One reason for the discrepancy is the potential for rapid discharges to occur after the hospital pharmacy has closed. This study has ensured a review of hospital practices and procedures.

\section{P 270 ANTICIPATORY PRESCRIBING: COMPARISON OF THE NUMBER OF DISCHARGE PRESCRIPTIONS WITH THE NUMBER OF FAST-TRACK DISCHARGES}

Grace Khoo, ${ }^{1}$ Andrew Dickman, ${ }^{1}$ Tracey Morris, ${ }^{2}$ Emma Montgomery, ${ }^{1}$ Andrea Whitfield'. 'Blackpool Teaching Hospitals NHS Foundation Trust, Blackpool, UK; ${ }^{2}$ Trinity Hospice, Blackpool 